\title{
Drug Therapy during Pregnancy
}

\author{
Dongmei Sun ${ }^{1}$, Janine Hutson ${ }^{1}$, and Facundo Garcia-Bournissen ${ }^{1}$ \\ ${ }^{1}$ Western University Schulich School of Medicine and Dentistry
}

May 4, 2020

Drug therapy in pregnancy may cause anxiety to both physicians and patients due to uncertainty regarding dosing and safety. Nevertheless, the need for medication is high given that maternal chronic illnesses such as hypertension or diabetes are on the rise ${ }^{1}$. In a national study in the United States over 33 years, the prevalence of prescription medication use by pregnant women in the first trimester increased by $62.5 \%$ between the first 2 years of the study and the last 2 years ${ }^{2}$. Furthermore, the average number of medications used anytime in pregnancy has increased two-fold ${ }^{2}$. More recent research from different countries has found that medications are widely used in pregnancy, with the prevalence of using at least one drug ranging from 60 to 90 percent, excluding vitamins and minerals ${ }^{3-5}$. Prescription of drugs with potential teratogenicity has also increased in pregnancy, including folate antagonists or angiotensin converting enzyme inhibitors ${ }^{3,4}$. This suggests that many women go into pregnancy with chronic conditions that require medications, including those may pose some degrees of risk to the fetus, since the maternal benefit may outweigh the risk or if the pregnancy is unplanned. Successful treatment of pregnant patients requires a correct diagnosis and providing treatment that not only is effective but also balances risks and benefits, as maternal health is the best defense for fetal health. However, concomitant health conditions, and the intrinsic complex physiological changes associated to pregnancy can make prescribing in this population a particularly challenging balancing act. Very few drugs have specific pregnancy dosing regimens supported by scientific evidence in spite of well-known pharmacokinetics changes during pregnancy affecting a large proportion of medications (Table 1$)^{6-10}$. Data on drug efficacy and safety is sorely lacking for pregnant women, even for drugs that have been available for decades. Pregnant patients are commonly excluded from clinical trials during the drug development process due to ethical and safety concerns, which implies that the majority of drug therapy data in pregnancy have been extrapolated from males and to a much lesser extent, from non-pregnant females ${ }^{11}$. However, using extrapolated data in pregnancy has a number of major drawbacks such as that the extrapolation commonly fails to account for the changes in drug metabolism related to pregnancy ${ }^{6}$. Multiple physiological changes in pregnancy, including those affecting pharmacokinetics, must be considered when prescribing. Furthermore, these changes can also be affected by genetic variability ${ }^{10}$. Some drugs need to be used at higher doses in pregnancy due to increasing metabolic demand. A classic example is thyroid hormone, which requires a 30 to 45 percent higher dose in order to maintain euthyroid state due to limited compensation in pregnant patients with underlying thyroid disease ${ }^{12}$. One of the biggest physiologic changes in pregnancy is the expansion of plasma volume by approximately 50 percent due to hormone-mediated vasodilation, leading to activation of the renin-angiotensin-aldosterone system ${ }^{10}$. This change results in and increased volume of distribution for hydrophilic drugs and reduced peak concentrations ${ }^{6}$. Increased glomerular filtration rate in pregnancy also increases elimination of renally cleared drugs; a classic example of this effect is lithium ${ }^{13}$. Changes in hepatic enzyme activity in pregnancy, including upregulation of cytochrome P450 and glucuronidation, are another cause of increased metabolism and elimination of drugs ${ }^{14}$. Increased metabolism of lamotrigine by glucuronidation results in low drug levels in pregnancy and this can be further affected by polymorphism of UDP-glucuronosyltransferases ${ }^{15}$. One strategy to address changes in drug metabolism is to use therapeutic drug monitoring (TDM) with blood levels; however, TDM analysis is not readily available for the majority of 
drugs used in pregnancy, limiting the usefulness of this strategy in clinical practice. Furthermore, lower total drug levels do not necessarily translate to less free drug due to volume expansion in pregnancy leading to relative hypoalbuminemia ${ }^{6}$. For some drugs, many clinicians modify the dosing regimen on their own based on limited pharmacokinetic data. For example, labelatol, a common antihypertensive used in pregnancy, has a half-life of only $1.7 \pm 0.27$ hours in patients in the 3rd trimester of pregnancy, compared to 6-8 hours outside of pregnancy ${ }^{16}$. This difference leads many clinicians to prescribe labelatol three times per day based on patient response instead of twice-per-day as per drug monograph ${ }^{16}$. However, there is considerable variation among clinicians due to lack of scientific evidence. For example, in patients diagnosed with acute pulmonary embolism, twice-per-day dosing and once-per-day dosing of low molecular weight heparin are both commonly used $^{17}$. Enoxaparin once-per-day versus twice-per-day in a population pharmacokinetics study both achieved target plasma concentration in pregnancy ${ }^{18}$; however, once daily regimen has not been universally adopted in clinical practice and no similar data are available for other low molecular weight heparins. Pregnancy is also a vulnerable period for the occurrence of cardiac events. In particular consideration must be given to patients known to have long QT syndrome or on QT prolonging drugs. The adrenergic nature of labor and delivery may lead to catecholaminergic polymorphic ventricular tachycardia in these patients. Furthermore, patients with congenital long QT syndrome are also at risk during a nine-month post-partum period ${ }^{19}$. Treatment with beta-blockers are the mainstay of therapy during pregnancy and in the post-partum period ${ }^{19}$. There is a lack of data for therapeutics that may be urgently needed intrapartum that may prolong the QT interval in susceptible patients. For example, oxytocin and carbetocin are both known to prolong the QT interval but may be required for prevention or treatment of postpartum hemorrhage ${ }^{20,21}$. Clinical guidelines for using oxytocin for augmentation or induction of labour are lacking and some obstetricians may choose to do an elective Caesarian section to avoid prolonged oxytocin in these patients although this is not evidence based due to lack of studies. In addition to the challenges in proper prescribing in pregnancy, the information on teratogenicity of drugs is also limited. Management of the care of a pregnant patient must balance the benefits of treating the maternal medical condition with possible adverse effects on the fetus. Clinicians must rely on animal data, data from pregnancy registries, and published case control studies and case reports to make these risk/benefit assessments. These resources are by no means ideal as results are confounded by recall bias, selection bias, and inconsistency, as well as lack of ability to extrapolate safety between species ${ }^{22}$. Other than a few specific drugs with clear evidence of harm, many drugs have limited information, leading to variable practice among clinicians and inconsistent information provided to patients. For example, while angiotensinconverting enzyme inhibitor induced fetopathy has been described since the 1990s and accepted by medical community consistently ${ }^{23}$, the risk of maternal corticosteroid use with increased risk of cleft lip and palate has not been consistent among studies ${ }^{24,25}$. In addition, long term corticosteroid use is also linked to increased risk of preeclampsia which needs to be taken into consideration for treatment and monitoring ${ }^{26}$. The US Food and Drug Administration recognized the limitation of the prior FDA classifications for medication use in pregnancy (A, B, C, D, X category system). Thus, the "Pregnancy and Lactation Labeling Rule" went into effect by the FDA in June 2015, requesting manufacturers to provide available information regarding risks in pregnancy and lactation in a narrative summary; however, according to a recent survey, less than 50 percent of prescribers were aware of this change, while more than half deemed the narrative summary not helpful ${ }^{27}$. Lack of quality data is one of the barriers identified in this survey. In addition to the rating systems used in various jurisdictions, an evidence-based medicine classification system has been developed by toxicologists, which can be used to assist clinical decision making. Unfortunately, all of the currently available systems are of limited utility due to reliance on small studies and inability to be updated frequently with new data $^{22}$. Since the thalidomide story in the 1960s, much advancement has been made in medical therapy in pregnant women. However there remains a significant knowledge gap in pharmacological information which may expose patients to either toxicity or under treatment with reduced efficacy. In addition, numerous commonly used medications lack concrete data on teratogenicity. Supported by the NIH, the Pediatric Trials Network is currently conducting a multicentre trial studying the pharmacokinetics and safety of commonly used drugs in lactating women and breastfeeding infants in North America (NCT03511118); similar studies in pregnancy are also warranted to guide appropriate dosing. Long term data on children with fetal exposure for developmental toxicity are also urgently needed, and while some data for newer medications is systematically 
collected by international registries, in general information on fetal risks of maternal exposures is limited to incidental data obtained from accidental exposures. Finally, making all up-to-date information readily available to clinicians should also be a priority.

\section{References}

1. Sun AJ, Li S, Zhang CA, Jensen TK, Lindahl-Jacobsen R, Eisenberg ML. Parental comorbidity and medication use in the USA: a panel study of 785000 live births. Hum Reprod.Mar 182020.

2. Mitchell AA, Gilboa SM, Werler MM, et al. Medication use during pregnancy, with particular focus on prescription drugs: 1976-2008. American journal of obstetrics and gynecology. Jul 2011;205(1):51 e51-58.

3. Ventura M, Maraschini A, D'Aloja P, et al. Drug prescribing during pregnancy in a central region of Italy, 2008-2012. BMC Public Health. May 15 2018;18(1):623.

4. Engeland A, Bjorge T, Klungsoyr K, Hjellvik V, Skurtveit S, Furu K. Trends in prescription drug use during pregnancy and postpartum in Norway, 2005 to 2015.Pharmacoepidemiology and drug safety. Sep 2018;27(9):995-1004.

5. Lutz BH, Miranda VIA, Silveira MPT, et al. Medication Use among Pregnant Women from the 2015 Pelotas (Brazil) Birth Cohort Study. International journal of environmental research and public health. Feb 5 2020;17(3).

6. Koren G, Pariente G. Pregnancy- Associated Changes in Pharmacokinetics and their Clinical Implications.Pharmaceutical research. Feb 12 2018;35(3):61.

7. Yerby MS, Friel PN, McCormick K, et al. Pharmacokinetics of anticonvulsants in pregnancy: alterations in plasma protein binding. Epilepsy research. Apr 1990;5(3):223-228.

8. Prevost RR, Akl SA, Whybrew WD, Sibai BM. Oral nifedipine pharmacokinetics in pregnancy-induced hypertension. Pharmacotherapy. 1992;12(3):174-177.

9. Philipson A. Pharmacokinetics of ampicillin during pregnancy. J Infect Dis. Sep 1977;136(3):370-376.

10. Blackburn S. Chapter 9. Cardiovascular System. Maternal, Fetal, \& Neonatal Physiology . 5th ed. London, United Kingdom: Elsevier; 2018:251-259.

11. Huybrechts KF, Bateman BT, Hernandez-Diaz S. Use of real-world evidence from healthcare utilization data to evaluate drug safety during pregnancy.Pharmacoepidemiology and drug safety. Jul 2019;28(7):906922.

12. Alexander EK, Pearce EN, Brent GA, et al. 2017 Guidelines of the American Thyroid Association for the Diagnosis and Management of Thyroid Disease During Pregnancy and the Postpartum. Thyroid. Mar 2017;27(3):315-389.

13. Wesseloo R, Wierdsma AI, van Kamp IL, et al. Lithium dosing strategies during pregnancy and the postpartum period. The British journal of psychiatry : the journal of mental science. Jul 2017;211(1):31-36.

14. Betcher HK, George AL, Jr. Pharmacogenomics in pregnancy. Seminars in perinatology. Jan 25 2020:151222.

15. Milosheska D, Lorber B, Vovk T, Kastelic M, Dolzan V, Grabnar I. Pharmacokinetics of lamotrigine and its metabolite N-2-glucuronide: Influence of polymorphism of UDP-glucuronosyltransferases and drug transporters. British journal of clinical pharmacology. Aug 2016;82(2):399-411.

16. Rogers RC, Sibai BM, Whybrew WD. Labetalol pharmacokinetics in pregnancy-induced hypertension. American journal of obstetrics and gynecology. Feb 1990;162(2):362-366.

17. Bates SM, Rajasekhar A, Middeldorp S, et al. American Society of Hematology 2018 guidelines for management of venous thromboembolism: venous thromboembolism in the context of pregnancy. Blood advances. Nov 27 2018;2(22):3317-3359. 
18. Patel JP, Green B, Patel RK, Marsh MS, Davies JG, Arya R. Population pharmacokinetics of enoxaparin during the antenatal period. Circulation. Sep 24 2013;128(13):1462-1469.

19. Garg L, Garg J, Krishnamoorthy P, et al. Influence of Pregnancy in Patients With Congenital Long QT Syndrome. Cardiology in review. Jul/Aug 2017;25(4):197-201.

20. Clunies-Ross N, Roston TM, Taylor J, et al. The Effect of Carbetocin Dose on Transmural Dispersion of Myocardial Repolarization in Healthy Parturients Scheduled for Elective Cesarean Delivery Under Spinal Anesthesia: A Prospective, Randomized Clinical Trial. Anesthesia and analgesia. Mar 52020.

21. Liou SC, Chen C, Wong SY, Wong KM. Ventricular tachycardia after oxytocin injection in patients with prolonged Q-T interval syndrome-report of two cases. Acta anaesthesiologica Sinica. Mar 1998;36(1):49-52.

22. Wilmer E, Chai S, Kroumpouzos G. Drug safety: Pregnancy rating classifications and controversies. Clinics in dermatology. May-Jun 2016;34(3):401-409.

23. Barr M, Jr., Cohen MM, Jr. ACE inhibitor fetopathy and hypocalvaria: the kidney-skull connection. Teratology. Nov 1991;44(5):485-495.

24. Carmichael SL, Shaw GM. Maternal corticosteroid use and risk of selected congenital anomalies. American journal of medical genetics. Sep 17 1999;86(3):242-244.

25. Czeizel AE, Rockenbauer M. Population-based case-control study of teratogenic potential of corticosteroids. Teratology. Nov 1997;56(5):335-340.

26. Cai E, Czuzoj-Shulman N, Abenhaim HA. Maternal and fetal outcomes in pregnancies with long-term corticosteroid use. The journal of maternal-fetal $\& 3$ neonatal medicine : the official journal of the European Association of Perinatal Medicine, the Federation of Asia and Oceania Perinatal Societies, the International Society of Perinatal Obstet. Aug 20 2019:1-8.

27. Namazy J, Chambers C, Sahin L, et al. Clinicians' Perspective of The New Pregnancy and Lactation Labeling Final Rule (PLLR): Results from an AAAAI/FDA Survey. The journal of allergy and clinical immunology. In practice. Feb 182020.

\section{Hosted file}

Table.docx available at https://authorea.com/users/316900/articles/447001-drug-therapy-duringpregnancy 